\title{
Internet-based Cognitive-behavioral Writing Therapy for Reducing Posttraumatic Stress After Severe Sepsis in Patients and Their Spouses (REPAIR): Results of a Randomized Controlled Trial
}

\section{Romina Gawlytta}

Jena University Hospital

Miriam Kesselmeier

Jena University Hospital

André Scherag

Jena University Hospital

Helen Niemeyer

Freie Universität Berlin

Maria Böttche

Freie Universität Berlin

Christine Knaevelsrud

Freie Universität Berlin

Jenny Rosendahl ( $\nabla$ jenny.rosendahl@med.uni-jena.de)

Institute of Psychosocial Medicine, Psychotherapy and Psychooncology, Jena University Hospital, Jena, Germany 2 Integrated Research and Treatment Center, Center for Sepsis Control and Care (CSCC), Jena University Hospital, Jena, Germany https://orcid.org/0000-0001-7535-7571

\section{Research}

Keywords: Posttraumatic stress disorder, Internet-based cognitive-behavioral writing therapy, Critical illness, Sepsis, Post-intensive care syndrome

Posted Date: September 15th, 2020

DOI: https://doi.org/10.21203/rs.3.rs-74259/v1

License: (c) (1) This work is licensed under a Creative Commons Attribution 4.0 International License. Read Full License 


\section{Abstract}

Background: Posttraumatic stress disorder (PTSD) is a frequent long-term consequence of intensive care in both, patients and spouses, and there is a specific need of respective tailored intervention approaches. Aim of this trial was to investigate the efficacy, safety and applicability of internet-based cognitivebehavioral writing therapy (iCBT) for posttraumatic stress after intensive care for sepsis in patients and their spouses compared to a waitlist (WL) control group.

Methods: In a randomized-controlled, parallel group, superiority trial we included patients after intensive care ( $>5$ days) for sepsis and their spouses, if at least one of them had a presumptive PTSD diagnosis (PTSD Checklist $[P C L-5] \geq 33$ ). Dyads were randomized 1:1 either to the iCBT (including two internetbased writing assignments per week over a 5-week period) or to the WL control group. Primary outcome was pre-post change in PTSD symptom severity score (measured via the PCL-5) in participants with a presumptive PTSD diagnosis from randomization to $\sim 5$ weeks later.

Results: Twenty-five dyads were included representing 34 participants with presumptive PTSD diagnosis (patient and spouse: 9 dyads, patient only: 12, spouse only: 4). We did not observe evidence for a difference in the primary outcome for iCBT compared to WL in the confirmatory analysis according to the intention-to-treat principle. The mean difference in score point reductions between iCBT and WL was -0.96 ( $95 \%$ confidence interval, -5.88 to $3.97 ; p=0.703$ ), which was adjusted for pre-values of the PCL-5 in the generalized estimating equations model. No adverse events were reported. Participants confirmed the applicability of iCBT.

\section{Conclusions:}

We applied an iCBT to reduce PTSD symptoms after sepsis and intensive care, for the first time addressing both patients and their spouses. ICBT was applicable in the given population. We did not observe evidence for the efficacy of iCBT for the primary outcome of pre-post changes in PCL-5 PTSD symptom severity score. Due to the small sample size our trial should be regarded as a pilot study and findings remain preliminary. Nevertheless, our results can guide further research, which is needed to determine if modified approaches to post-intensive care PTSD may be more effective.

Trial registration: German Clinical Trials Register (DRKS00010676). Registered 14 June 2016, https://www.drks.de/drks_web/navigate.do? navigationld=trial.HTML\&TRIAL_ID=DRKS00010676

\section{Introduction}

Experience of intensive care could affect mental health of both patients and their partners. About every fifth patient and an equal proportion of spouses develop a posttraumatic stress disorder (PTSD) as a long-term consequence of treatment in the intensive care unit (ICU) [1-4]. Thus, PTSD has been considered as part of the post-intensive care syndrome (PICS) and PICS-family [5]. Research on post-ICU consequences revealed that mental health of patients and their spouses following ICU experiences are 
interrelated and that couples seem to react as a dyadic system to a life-threatening situation [6-9]. In the context of dyadic coping research, it has been suggested to use the term 'we-disease' to describe that both, the patient and his/her partner, face the illness "as a shared 'we-event' and a 'we-experience' rather than an individual problem of one partner requiring support from the other" (p. 595) [10]. The concept of 'we-disease' also implies that the treatment of mental distress associated with the illness should always include both partners as they both suffer but also have resources and can jointly contribute to the coping process $[6,10]$.

In the past few years, various intervention approaches have been developed to address PTSD in patients or family members that might be classified as interventions during ICU care to prevent PTSD or as interventions to treat PTSD in the long-term run. Preventive PTSD interventions usually consider a broad target group of ICU patients or family members at risk for post-ICU PTSD, e.g. [11-16]. Contrasting, PTSD treatment interventions are provided several months after ICU discharge and specifically address patients or family members with clinically significant PTSD symptoms and PTSD diagnosis, respectively, e.g. [17].

For the treatment of PTSD, clinical guidelines in general strongly recommend trauma-focused psychotherapy with cognitive-behavioral components of exposure and/or cognitive restructuring $[18,19]$. Implemented via the internet, cognitive-behavioral therapy can meet the specific needs of post-ICU patients by providing access for mobility-impaired patients or patients with speech and hearing difficulties [20].

The primary objective of the REPAIR (Reducing posttraumatic stress after severe sepsis in patients and their spouses) trial was to investigate the efficacy, safety and applicability of a therapist-guided internetbased cognitive-behavioral writing therapy (iCBT) for posttraumatic stress after intensive care for sepsis in patients and their spouses compared to a waitlist (WL) control group. Second, considering the interrelation of mental health between patients and partners, the study aimed at examining dyadic concordance in treatment effects, i.e. indirect effects of the treatment in the respective spouse of the treated participant.

\section{Materials And Methods}

\section{Study design and setting}

The REPAIR trial was a randomized, controlled, open-label, parallel group superiority trial conducted at the Jena University Hospital (JUH), Germany. Dyads of a post-ICU patient and his/her spouse were randomly assigned to either iCBT or to a WL control group (allocation ratio 1:1) with the dyad being the unit of randomization. Allocation was performed centrally using computer-generated random numbers provided by an independent center for clinical trials at the JUH and stratified by the occurrence of PTSD symptoms within the dyad of the post-ICU patient and the spouse (stratum 1: both post-ICU patient and spouse with PTSD, stratum 2: post-ICU patient with PTSD and spouse without PTSD, stratum 3: post-ICU patient without PTSD and spouse with PTSD). The trial was approved by the ethics committee of the Friedrich- 
Schiller University Jena, Germany (number 4777-04/16, 11 May 2016) and registered in the German Clinical Trials Register (DRKS; number DRKS00010676). The study protocol has been published previously [20].

\section{Participants}

We included dyads (each member $\geq 18$ years) comprising a former patient, who was treated for sepsis on an ICU for more than five days and discharged from ICU more than one month ago, and his/her spouse (married or cohabited). A patient-spouse dyad was included if at least one of them presented with a presumptive PTSD diagnosis with regard to the life-threating event (see definition below). Reasons for exclusion on dyad-level were not having a spouse as well as acute psychosis, suicidal ideation, use of neuroleptics, not being fluent in German or ongoing psychotherapeutic treatment elsewhere of at least one dyad member. Participants were screened for eligibility in a telephone interview by using the PTSD checklist for DSM-5 (PCL-5) [21] and completed the Life Event Checklist for DSM-5 (LEC 5) [22]. Written informed consent was obtained by the patients and their spouses. In a second telephone contact, patients and their spouses with presumptive PTSD diagnosis according to PCL-5 completed the ClinicianAdministered PTSD Scale for DSM-5 (CAPS-5) [23] and the Structured Clinical Interview for DSM-IV (SCIDI) [24] conducted by a trained clinical psychologist (RG). Furthermore, participants were asked to provide medical data (e.g., length of intensive care, length of mechanical ventilation (if applicable) and time since ICU discharge).

\section{Intervention}

Participants allocated to the treatment condition participated in an iCBT targeted to the traumatic ICU situation. They completed two 50-minute internet-based writing assignments per week over a five-week period (10 essays in total). The treatment consisted of three modules: (I) resource-oriented biographical reconstruction (three essays), (II) in sensu trauma exposure sessions (four essays), and (III) cognitive reconstruction (three essays). After completion of each assignment, the therapist provided individual feedback and further writing instructions to the participant within one workday. Integrated in the third module, the treated participant received a supportive letter from his/her respective partner. Participants without clinically relevant PTSD symptoms (PCL-5<33) only completed the assessments and received psychoeducational information about mental health problems after traumatic events. Participants allocated to the WL control group also received iCBT after five weeks of waiting (duration of treatment), but without a supportive letter from their spouses. For details, we refer to the published study protocol [20].

\section{Outcomes}

Primary outcome was change in PTSD symptom severity score from baseline to the end of treatment/waiting time (about 5 weeks after randomization) measured via the German version of PCL-5 $[21,25]$ covering the four DSM-5 symptom clusters. A cut-off of 33 was used for a presumptive PTSD diagnosis [26]. A change of 10 points or more is regarded as clinically relevant [21]. 
Secondary efficacy outcomes were (a) symptoms of psychological distress, (b) relationship satisfaction, (c) health-related quality of life, and (d) remission at the end of treatment/waiting time. Safety endpoints were (i) the number of suicide alerts, (ii) the number of participants with a clinically relevant PCL-5 deterioration, and (iii) the percentage of participants leaving the study early (during treatment phase) due to any reason. An additional secondary endpoint was dyadic concordance in treatment effects (in terms of PCL-5). Psychological distress was measured using the German version of the Brief Symptom Inventory-18 including subscales of anxiety, depression, and somatization (BSI-18) [27, 28]. Relationship satisfaction was assessed with the German version of the Relationship Assessment Scale (RAS) [29, 30]. The German version of the health questionnaire of the EuroQol group (EQ-5D-5L) [31, 32] was used to measure health-related quality of life on five dimensions (mobility, self-care, usual activities, pain/discomfort and anxiety/depression). Utility values of EQ-5D-5L were derived according to Ludwig et al. [32]. Remission was only considered for participants diagnosed with PTSD before treatment/waiting period. For those, remission was defined as being free of PTSD diagnosis after the intervention/waiting period. PTSD was diagnosed by a trained clinical psychologist (RG) as described above (for details, Supplemental Digital Content 1).

\section{Sample size}

The sample size calculation was based on Student's t-test for a parametric two-group comparison, even though more complex models that address the clustering would be used for the confirmatory analysis. In accordance with a recent meta-analysis [33], we considered effect sizes quantified by Cohen's $d$ of 0.95 as realistic. To detect differences between the treatment groups at a two-sided significance level of 0.05 with a comparison-wise power of 0.9 , a sample size of $2 \times 34$, i.e. 68 patient-spouse dyads, is required for the intention-to-treat (ITT) analysis. Assuming a dropout rate of $30 \%$, the total sample size would be 98 dyads. A higher power was chosen to address the fact that a more complex statistical analysis approach would be used.

\section{Statistical methods}

We rely on two populations. The dyad population includes randomized participants irrespective of their presumptive PTSD diagnosis. The PTSD population only comprises participants with a presumptive PTSD diagnosis. Primary and secondary efficacy/safety outcomes are analyzed in the PTSD population, while dyadic concordance is assessed in the dyad population.

The handling of missing data was predefined in the study protocol and/or the statistical analysis plan (SAP). Based on the expected high internal consistency of the scores, we substituted missing items with the mean of the provided items of the respective participant if $10 \%$ or fewer items were missing. We apply the intention-to-treat (ITT) and the per-protocol (PP) principle to both populations. In case of the perprotocol (PP) principle, we include randomized participants (with a presumptive PTSD diagnosis) who provided pre- ( $\mathrm{t} 0$ ) and post-treatment/waiting ( $\mathrm{t} 1$ ) information. In case of the ITT principle, we consider all randomized participants (with a presumptive PTSD diagnosis). Missing score values were replaced, stratified by intervention group and type of treatment (defined according to the strata for the 
randomization), according to best-case/worst-case substitution. We denote this data set as "primary analysis set". As additional sensitivity analysis that was not pre-specified in the study protocol, we used multiple imputation by chained equations (MICE) using fully conditional specification [34] (for details, Supplemental Digital Content 1).

Participant characteristics (dyad population) and outcomes (PTSD population) are summarized as absolute and relative frequencies for nominal variables or as medians together with the first and third quartile (Q1, Q3) for ordinal/continuous variables. Rough group comparisons were done by Fisher's exact test or Mann-Whitney-U test. For the primary outcome PCL- 5 change, we applied generalized estimating equation (GEE) modelling (independent variables: baseline PCL-5 value, treatment condition; cluster: dyad) in the primary analysis set. We performed several sensitivity analyses (ITT principle with MICE, PP principle, extension of the above defined GEE model by inclusion of further possible confounders as independent variables). For the secondary efficacy outcomes, we adapted the GEE modelling accordingly. For primary and secondary efficacy outcomes, model coefficients (adjusted mean differences or odds ratios (OR)) with $95 \%$ confidence intervals (Cls) and p-values are presented. In addition, we provide the corresponding between-group effect sizes (standardized mean differences, Cohen's $d$ ). For the safety outcomes, we provide absolute and relative frequencies from the PP population. Dyadic concordance in treatment effects (in terms of PCL-5) was assessed with Spearman correlation (together with the corresponding $95 \% \mathrm{Cls}$ ) independently from the treatment condition between post-ICU patients and his/her spouse in the dyad population according to both ITT and PP principle. We applied a two-sided significance level of 0.05 to the primary confirmatory analysis and did not correct for multiple testing otherwise as the other analyses were considered exploratory. We used R (version 3.6.0) for statistical analyses (for details, Supplemental Digital Content 1).

\section{Results}

\section{Participants}

Between February 2017 and January 2019, we received 57 enquiries from either a post-ICU patient or his/her spouse. After screening for eligibility 25 dyads were randomized, 12 to iCBT and 13 to WL (Fig. 1). Median age of the study participants was 55 years (Q1-Q3, 47-62). Thirty-four participants had a presumable PTSD diagnosis ( 9 dyads with affected post-ICU patient and spouse, 12 with post-ICU patient only, 4 with spouse only). Of those, 25 were initially diagnosed with PTSD in the clinical interview (iCBT: 14; WL: 11). Further characteristics of the participants are shown in Table 1 (for stratification by post-ICU patient/spouse, Supplemental Digital Content 2, Supplemental Table S1; for descriptive summary of the outcomes, Supplemental Tables S2 and S3). Of note, one participant dropped-out directly after randomization. For details on missing data and its impact/handling, we refer to Supplemental Digital Content 1. 
Table 1

Characteristics of participants - overall as well as stratified by treatment group.

\begin{tabular}{|c|c|c|c|c|}
\hline \multirow[t]{2}{*}{ Characteristic } & \multirow[t]{2}{*}{ Overall } & \multicolumn{2}{|c|}{ Treatment group } & \multirow{2}{*}{$\begin{array}{l}p- \\
\text { value }\end{array}$} \\
\hline & & iCBT & $\begin{array}{l}\text { WL } \\
\text { control }\end{array}$ & \\
\hline Male sex; $n(\%)$ & $26(52.0)$ & $12(50.0)$ & $14(53.8)$ & 1.000 \\
\hline Age, in years; median (Q1, Q3) & $\begin{array}{l}55(47 \\
62)\end{array}$ & $\begin{array}{l}56(52 \\
64)\end{array}$ & $\begin{array}{l}54(46, \\
59)\end{array}$ & 0.101 \\
\hline \multicolumn{5}{|l|}{ Among post-ICU patients ${ }^{\star *}$} \\
\hline Time since ICU treatment, in years; median (Q1, Q3) & $\begin{array}{l}\text { 1.8 (1.1, } \\
3.7)\end{array}$ & $\begin{array}{l}1.9(1.2 \\
4.6)\end{array}$ & $\begin{array}{l}1.6(1.0 \\
2.0)\end{array}$ & 0.231 \\
\hline Duration of ICU treatment, in days; median (Q1, Q3) & $\begin{array}{l}21(13, \\
40)\end{array}$ & $\begin{array}{l}28(12, \\
42)\end{array}$ & $\begin{array}{l}21(13, \\
28)\end{array}$ & 0.662 \\
\hline Mechanical ventilation & & & & 1.000 \\
\hline Yes; $n(\%)$ & $18(72.0)$ & $9(75.0)$ & $9(69.2)$ & \\
\hline No; $n(\%)$ & $5(20.0)$ & $2(16.7)$ & $3(23.1)$ & \\
\hline Not specified; $n(\%)$ & $2(8.0)$ & $1(8.3)$ & $1(7.7)$ & \\
\hline $\begin{array}{l}\text { Duration of mechanical ventilation among ventilated } \\
\text { patients, in days; median }(\mathrm{Q} 1, \mathrm{Q} 3)^{\star \star \star \star}\end{array}$ & $\begin{array}{l}24(16, \\
28)\end{array}$ & $\begin{array}{l}28(28 \\
35)\end{array}$ & $\begin{array}{l}18(8, \\
23)\end{array}$ & 0.048 \\
\hline College or university degree; $n(\%)$ & $17(34.0)$ & $7(29.2)$ & $10(38.5)$ & 0.559 \\
\hline Pre-existing mental disorder (prior to sepsis); $n$ (\%) & $16(32.0)$ & $9(37.5)$ & $7(26.9)$ & 0.547 \\
\hline \multicolumn{5}{|l|}{ Treatment of pre-existing mental disorder } \\
\hline Prior to sepsis; $n(\%)$ & $15(30.0)$ & $8(33.3)$ & $7(26.9)$ & 0.760 \\
\hline
\end{tabular}

The numbers are based on the dyad population. Overall, there are 25 dyads -12 dyads in the iCBT group and 13 dyads in the WL control group. Note that each dyad comprises one post-ICU patient and one spouse. Characteristics are summarized as median with first and third quartile (Q1, Q3) or as absolute (n) and relative frequency (\%). P-values are derived from Mann-Whitney-U test and Fisher's exact test, respectively, while excluding patients with missing (including non-specified) information on the respective characteristic. Abbreviations: $\mathrm{iCBT}$, internet-based cognitive-behavioral writing therapy; ICU, intensive care unit; PTSD, post-traumatic stress disorder; WL, waitlist.

* percentage refers to dyad; ${ }^{* \star}$ percentage refers to former ICU patient; ${ }^{* \star}$ percentage refers to partner; ${ }^{\star \star \star \star *}$ missing for 9 patients (iCBT group: 6, WL control group: 3 


\begin{tabular}{|c|c|c|c|c|}
\hline \multirow[t]{2}{*}{ Characteristic } & \multirow[t]{2}{*}{ Overall } & \multicolumn{2}{|c|}{ Treatment group } & \multirow{2}{*}{ value } \\
\hline & & iCBT & $\begin{array}{l}\text { WL } \\
\text { control }\end{array}$ & \\
\hline Post sepsis; $n$ (\%) & $6(12.0)$ & $4(16.7)$ & $2(7.7)$ & 0.409 \\
\hline \multicolumn{5}{|l|}{ Presumptive PTSD diagnosis } \\
\hline Post-ICU patient only; $n(\%)^{\star \star}$ & $12(48.0)$ & $6(50.0)$ & $6(46.2)$ & 1.000 \\
\hline Spouse only; $n(\%)^{* \star *}$ & $4(16.0)$ & $2(16.7)$ & $2(15.4)$ & 1.000 \\
\hline Both dyad members; $\mathrm{n}(\%)^{*}$ & $9(36.0)$ & $4(33.3)$ & $5(38.5)$ & 1.000 \\
\hline \multicolumn{5}{|l|}{ Relationship } \\
\hline Duration, in years; median $(\mathrm{Q} 1, \mathrm{Q} 3)^{*}$ & $\begin{array}{l}22.2 \\
(16.2 \\
32.9)\end{array}$ & $\begin{array}{l}24.5 \\
(19.1 \\
34.6)\end{array}$ & $\begin{array}{l}21.8 \\
(12.5 \\
29.4)\end{array}$ & 0.414 \\
\hline Marital status: married; $n(\%)^{*}$ & $21(84.0)$ & $10(83.3)$ & $11(84.6)$ & 1.000 \\
\hline \multicolumn{5}{|c|}{$\begin{array}{l}\text { The numbers are based on the dyad population. Overall, there are } 25 \text { dyads }-12 \text { dyads in the iCBT } \\
\text { group and } 13 \text { dyads in the WL control group. Note that each dyad comprises one post-ICU patient and } \\
\text { one spouse. Characteristics are summarized as median with first and third quartile (Q1, Q3) or as } \\
\text { absolute (n) and relative frequency (\%). P-values are derived from Mann-Whitney-U test and Fisher's } \\
\text { exact test, respectively, while excluding patients with missing (including non-specified) information on } \\
\text { the respective characteristic. Abbreviations: iCBT, internet-based cognitive-behavioral writing therapy; } \\
\text { ICU, intensive care unit; PTSD, post-traumatic stress disorder; WL, waitlist. }\end{array}$} \\
\hline \multicolumn{5}{|c|}{ 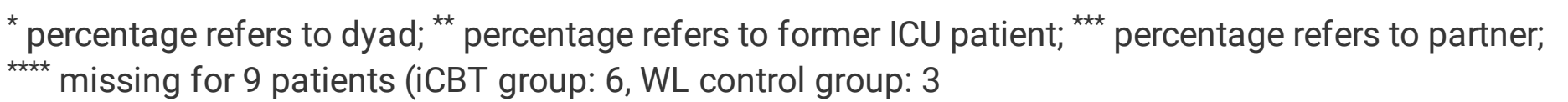 } \\
\hline
\end{tabular}

\section{Primary outcome}

Individual, time-dependent PCL-5 curves are shown in Fig. 2. In the primary analysis set, we did not observe evidence for differences between groups in the primary outcome. The adjusted mean difference in PCL-5 score change was -0.96 (95\% Cl, -5.88 to $3.97 ; p=0.703$; Table 2) when comparing iCBT to WL. Sensitivity analyses also showed no evidence for differences in PCL- 5 change between the iCBT and the WL control group (ITT with MICE: $4.01 ; 95 \% \mathrm{Cl},-1.89$ to $9.91 ; p=0.181 ; \mathrm{PP}: 2.40 ; 95 \% \mathrm{Cl},-2.29$ to $7.08 ; p=$ 0.316 ; Table 2). The corresponding between-group effect sizes varied between $-0.14(95 \% \mathrm{Cl},-0.81$ to $0.54)$ and 0.48 ( $95 \% \mathrm{Cl},-0.21$ to 1.16$)$ (Supplemental Table S9). The extended multivariable models revealed similar results (Supplemental Digital Content 2, Supplemental Table S4). 
Table 2

Results for PCL-5 (PTSD Checklist for DSM-5) change from multivariable generalized estimating equation (GEE) modelling.

\begin{tabular}{|c|c|c|}
\hline Variable & Mean difference $(95 \% \mathrm{Cl})$ & $p$-value \\
\hline \multicolumn{3}{|c|}{ ITT (best-case/worst-case) } \\
\hline iCBT [ref.: no] & $-0.96(-5.88,3.97)$ & 0.703 \\
\hline Baseline value (t0) & $0.09(-0.05,0.23)$ & 0.225 \\
\hline \multicolumn{3}{|l|}{ ITT (MICE) } \\
\hline iCBT [ref.: no] & $4.01(-1.89,9.91)$ & 0.181 \\
\hline Baseline value (t0) & $0.16(-0.02,0.33)$ & 0.078 \\
\hline \multicolumn{3}{|l|}{$P P$} \\
\hline iCBT [ref.: no] & $2.40(-2.29,7.08)$ & 0.316 \\
\hline Baseline value (t0) & $0.10(-0.03,0.23)$ & 0.123 \\
\hline \multicolumn{3}{|c|}{$\begin{array}{l}\text { Model coefficients (mean difference) together with } 95 \% \text { confidence intervals (Cls) and p-values are } \\
\text { provided. Results from both intention-to-treat (ITT) approaches (best-case/worst-case as main } \\
\text { analysis, multiple imputation by chained equations (MICE) as sensitivity analysis) and the per- } \\
\text { protocol (PP) analysis (sensitivity analysis) are provided. For binary variables, the reference category } \\
\text { (ref.) is provided. Note that there were five participants in the iCBT group and none in the waitlist } \\
\text { control group with missing information (missing PCL-5 change: } 5 \text {, missing baseline value: } 1 \text {; } \\
\text { Supplemental Digital Content 1, Additional Figures A3 and A4). Abbreviations: iCBT, internet-based } \\
\text { cognitive-behavioral writing therapy; t0, time point at beginning of intervention/waitlist period. }\end{array}$} \\
\hline
\end{tabular}

\section{Secondary efficacy outcomes}

In the primary analysis set, we explored that iCBT may lead to a larger RAS change than waiting (1.11; $95 \% \mathrm{Cl}, 0.64$ to $1.57 ; p<0.001$; Supplemental Digital Content 2, Supplemental Table S5). The corresponding between-group effect sizes for RAS change was large with 1.67 (95\% Cl, 0.89 to 2.45; Supplemental Digital Content 2, Supplemental Table S9). This observation was consistent across all sensitivity analyses (Supplemental Digital Content 2, Supplemental Table S5). For all other secondary efficacy outcomes, we did not observe evidence for an association between score changes and iCBT in the primary analysis set (Supplemental Digital Content 2, Supplemental Tables S6 to S8) with corresponding effect sizes of $0.04(95 \% \mathrm{Cl},-0.64$ to 0.71$)$ for BSI-18 and $0.25(95 \% \mathrm{Cl},-0.42$ to 0.93$)$ for EQ-5D-5L (Supplemental Table S9). Among patients with initial PTSD diagnosis (according to CAPS-5), remission rates were $64 \%$ after iCBT and $27 \%$ after waiting. Of note, $95 \%$ Cls for the iCBT effect for remission are wide in both ITT and, particularly, in the PP analyses; a smaller number of participants was considered in theses analyses as only participants with a presumptive PTSD diagnosis at baseline were included (Supplemental Digital Content 2, Supplemental Table S6).

\section{Safety and applicability}


Overall, there were five suicide alerts. All of them were clarified in immediate therapeutic contacts by telephone (see [20] for a description of safety management). Three revealed to be false alarms, two were caused by reasons not related to the study and the suicidal ideations subsided quickly. During iCBT/waiting, there were no clinical relevant deteriorations in regard of the PCL-5 score. Seven participants prematurely terminated in the iCBT group and two during waiting time, respectively. All dropouts appeared for reasons other than study or treatment participation (for further details on the safety endpoints, Supplemental Digital Content 2, Supplemental Tables S10 and S11). In-depth interviews with participants after the treatment confirmed the applicability of the intervention. It was positively highlighted that iCBT met the specific needs of the patients and the spouses. In terms of feasibility, no major technical problems emerged and the internet literacy of the participants was sufficient to complete the treatment.

\section{Dyadic concordance in treatment effects}

No evidence for a correlation between the PCL-5 changes of post-ICU patients and those of his/her spouse could be observed - neither in case of only one dyad member nor in case both dyad members had a presumptive PTSD diagnosis (Supplemental Digital Content 2, Supplemental Table S12).

\section{Discussion}

Posttraumatic stress symptoms are a frequent, well-documented sequelae after intensive care, both in the patient and his/her spouse [1-4] and with dyadic concordance of mental impairments within the couple [6-9]. Previous studies have targeted PTSD prevention during hospital stay [11-16] and post-ICU interventions [17] with variable success. To our knowledge there is no previous study that evaluated an intervention involving both patients and spouses with the goal of reducing these PTSD symptoms. Consequently, we conducted a randomized-controlled trial testing the efficacy, safety and applicability of an iCBT for reducing PTSD symptoms in patients and their spouses.

We included 25 dyads resulting in 34 treated participants which however did not represent the planned sample size. With regard to our primary outcome, we could not observe evidence that iCBT led to a larger reduction of PTSD symptom severity than waiting. With regard to secondary outcomes, we explored a relatively larger increase of relationship satisfaction in the iCBT compared to the WL control group which merits confirmation in future studies. Contrary to our hypothesis, there was no evidence for dyadic concordance in any of the treatment effects.

Beyond efficacy, participating in iCBT was safe, as no adverse events such as suicidality or clinically relevant PTSD symptom deterioration occurred that were therapy-related. Although seven participants prematurely terminated in the iCBT group, all dropouts appeared for reasons other than study or treatment participation. Furthermore, participants confirmed the applicability of iCBT and the feasibility of the implementation. Hence, iCBT can be regarded as an applicable intervention in the particular population of post-ICU patients and their spouses. 
The need for ICU follow-up care to diagnose and treat PICS impairments after hospital discharge is apparent. Post-ICU patients show an increased utilization of outpatient specialist services, including psychiatric services, higher medication intake, and impaired quality of life [35]. Specialized post-ICU outpatient clinics could provide the necessary services specific to ICU survivors' healthcare needs [5], but are however not yet established nationwide in Germany. Internet-based treatment approaches like iCBT in the follow-up of ICU patients can be particularly helpful for physically impaired patients or patients living in considerable distance from the hospital or specialized outpatient care, regardless of whether they are cared for in an ICU follow-up clinic or not.

A key strength of our study is the involvement of both patients and spouses in the treatment of post-ICU posttraumatic stress. In this regard, we implemented writing a supportive letter to the respective spouse as a dyadic treatment component in the iCBT [20]. Including both partners in the study (though not always both were treated) might have led to in-depth communication about their individual traumatic experiences and its consequences to their partnership [36]. Considering the concept of 'we-disease', this might have contributed to a strengthening of dyadic resources and coping efforts [10], finally affecting both partnership and individual well-being.

There are, however, several important limitations that may have affected the results. First, we did not achieve the planned sample size. Despite tremendous efforts and a significant extension of the recruitment period, we experienced serious problems in recruiting participants. We can only speculate about the reasons. As internet-delivered psychotherapy is not yet part of routine care in Germany, there might have been concerns and caveats about the practicability and safety of the intervention and the (primarily) elderly patients might be less open for such "new" approaches. Furthermore, there are no specialized post-ICU rehabilitation and outpatient ICU follow-up clinics in Germany, making it difficult to "find" and contact patients after hospital discharge. The small sample size has resulted in a lack of statistical power. Hence, our results should be regarded as preliminary and further trials are needed to prove the efficacy of iCBT in the context of post-ICU PTSD. Another problem emerged from missing data due to premature termination. To follow the ITT principle, we imputed missing data based on the bestcase/worst-case substitution as the most rigorous method. We further included sensitivity analyses applying multiple imputation and relying on the PP population. Note that there are differences in the assumptions of these approaches reflecting common challenges in dealing with missing data. Hence, our conclusions remain fraught with uncertainty. Finally, generalizability of our results is restricted to sepsis patients and their spouses from Germany.

\section{Conclusions}

In sum, we could not prove the efficacy of iCBT in contrast to waiting in patients and spouses after intensive care treated sepsis with a presumptive PTSD diagnosis. Explorations indicate that ICBT may lead to a larger increase of relationship satisfaction in post-ICU patients and spouses than waiting. We also demonstrated that iCBT is safe and applicable for both post-ICU patients and their spouses. The largest limitation of the REPAIR trial was the small sample size so that our trial should be regarded as a 
pilot study and findings remain preliminary. Future research could benefit by considering our findings and experiences in the planning of further tailored randomized-controlled trials.

\section{Abbreviations}

BSI-18

Brief Symptom Inventory-18

CAPS-5

Clinician-Administered PTSD Scale for DSM-5

$\mathrm{Cl}$

confidence interval

DSM-5

Diagnostic and Statistical Manual of Mental Disorders

EQ-5D-5L

health questionnaire of the EuroQol group - 5 levels

GEE

generalized estimating equation

LEC-5

Life Event Checklist for DSM-5

iCBT

internet-based cognitive-behavioral writing therapy

ITT

intention-to-treat

$\mathrm{JUH}$

Jena University Hospital

MICE

multiple imputation by chained equations

OR

odds ratio

PICS

post-intensive care syndrome

PCL-5

PTSD checklist for DSM-5

PP

per protocol

PTSD

posttraumatic stress disorder

RAS

Relationship Assessment Scale

REPAIR 
Reducing posttraumatic stress after severe sepsis in patients and their spouses

SAP

statistical analysis plan

SCID

Structured Clinical Interview for DSM-IV

WL

waitlist

\section{Declarations}

\section{Funding}

This study was funded by the German Federal Ministry of Education and Research (BMBF), grant number 01E01502. MK and AS were supported by the Integrated Research and Treatment Center - Center for Sepsis Control and Care (CSCC) at the Jena University Hospital, funded by the BMBF (grant number 01E01502).

\section{Acknowledgments}

We would like to kindly thank Uwe Ziegler and Cornelia Baumgart for their continuous IT support and Carolin Fleischmann-Struzek, Heike Romeike, and Ulrike Redlich for their helpful support in recruitment of participants.

\section{Author information}

Jenny Rosendahl and Christine Knaevelsrud contributed equally to this work.

\section{Affiliations}

Institute of Psychosocial Medicine, Psychotherapy and Psychooncology, Jena University Hospital, Jena, Germany: Romina Gawlytta, Jenny Rosendahl

Integrated Research and Treatment Center, Center for Sepsis Control and Care (CSCC), Jena University Hospital, Jena, Germany: Romina Gawlytta, Miriam Kesselmeier, André Scherag, Jenny Rosendahl Institute of Medical Statistics, Computer and Data Sciences, Jena University Hospital, Jena, Germany: Miriam Kesselmeier, André Scherag

Department of Clinical Psychological Intervention, Freie Universität Berlin, Berlin, Germany: Helen Niemeyer, Maria Böttche, Christine Knaevelsrud

Center ÜBERLEBEN, Berlin, Germany: Maria Böttche

\section{Corresponding author}


Correspondence to Jenny Rosendahl, email: jenny.rosendahl@med.uni-jena.de

\section{Contributions}

JR and CK conceived the study. CK, MB and HN developed the iCBT treatment manual. AS a priori conducted the power analysis and defined the statistical methods. MK was in charge of the statistical analysis and interpretation of the data. RG recruited patients, collected the data for the study and conducted all clinical interviews. MB and HN carried out the treatment of the participants. JR, MK and RG drafted the paper. All authors critically reviewed the manuscript. All authors read and approved the final version of the manuscript.

\section{Ethics declarations}

\section{Ethics approval and consent to participate}

The trial was approved by the ethics committee of the Friedrich-Schiller University Jena, Germany (number 4777-04/16, 11 May 2016) and written informed consent was obtained from the patient and his/her spouse.

\section{Consent for publication}

Not applicable.

\section{Competing interests}

None declared.

\section{References}

1. Parker AM, Sricharoenchai T, Raparla S, Schneck KW, Bienvenu OJ, Needham DM. Posttraumatic stress disorder in critical illness survivors: a metaanalysis. Crit Care Med. 2015;43(5):1121-9.

https://doi.org/10.1097/CCM.0000000000000882 2. Hickman RL Jr, Douglas SL. Impact of chronic critical illness on the psychological outcomes of family members. AACN Adv Crit Care. 2010;21(1):80-91. https://doi.org/10.1097/NCl.0b013e3181c930a3 3. Davidson JE, Jones C, Bienvenu OJ. Family response to critical illness: postintensive care syndrome-family. Crit Care Med. 2012;40(2):618-624.

https://doi.org/10.1097/CCM.0b013e318236ebf9 4. Petrinec AB, Daly BJ. Post-Traumatic Stress Symptoms in Post-ICU Family Members: Review and Methodological Challenges. West $J$ Nurs Res. 2016;38(1):57-78. https://doi.org/10.1177/0193945914544176 5. Needham DM, Davidson J, Cohen H, Hopkins RO, Weinert C, Wunsch $\mathrm{H}$, et al. Improving long-term outcomes after discharge from intensive care unit: report from a stakeholders' conference. Crit Care Med. 2012;40(2):502-509. https://doi.org/10.1097/CCM.0b013e318232da75 6. Rosendahl J, Brunkhorst FM, Jaenichen D, Strauss B. Physical and mental health in patients and spouses after intensive care of severe sepsis: a dyadic perspective on long-term sequelae testing the Actor-Partner Interdependence Model. Crit Care Med. 
2013;41(1):69-75. https://doi.org/10.1097/CCM.0b013e31826766b0 7. Fumis RR, Ranzani OT, Martins PS, Schettino G. Emotional disorders in pairs of patients and their family members during and after ICU stay. PLoS One 2015;10(1):e0115332. https://doi.org/10.1371/journal.pone.0115332 8. Wintermann GB, Weidner K, Strauß B, Rosendahl J, Petrowski K. Predictors of posttraumatic stress and quality of life in family members of chronically critically ill patients after intensive care. Ann Intensive Care 2016;6(1):69. https://doi.org/10.1186/s13613-016-0174-0 9. Wintermann GB, Petrowski K, Weidner K, Strauß B, Rosendahl J. Impact of post-traumatic stress symptoms on the health-related quality of life in a cohort study with chronically critically ill patients and their partners: age matters. Crit Care 2019;23(1):39. https://doi.org/10.1186/s13054-019-2321-0 10. Bodenmann G. Illness and dyadic coping. In: Wright JD, editor. International Encyclopedia of the Social \& Behavioral Sciences (Second Edition). Oxford: Elsevier; 2015. p. 593-5. 11. Peris A, Bonizzoli M, lozzelli D, Migliaccio ML, Zagli G, Bacchereti A, et al. Early intraintensive care unit psychological intervention promotes recovery from post traumatic stress disorders, anxiety and depression symptoms in critically ill patients. Crit Care. 2011;15(1):R41.

https://doi.org/10.1186/cc10003 12. Wade DM, Mouncey PR, Richards-Belle A, Wulff J, Harrison DA, Sadique MZ, et al. Effect of a Nurse-Led Preventive Psychological Intervention on Symptoms of Posttraumatic Stress Disorder Among Critically III Patients: A Randomized Clinical Trial. JAMA. 2019;321(7):665-675. https://doi.org/10.1001/jama.2019.0073 13. White DB, Angus DC, Shields A-M, Buddadhumaruk P, Pidro C, Paner C, et al. A Randomized Trial of a Family-Support Intervention in Intensive Care Units. New England Journal of Medicine. 2018;378(25):2365-75. https://doi.org/ 10.1056/NEJMoa1802637 14. Amass TH, Villa G, Omahony S, Badger JM, McFadden R, Walsh T, et al. Family Care Rituals in the ICU to Reduce Symptoms of Post-Traumatic Stress Disorder in Family Members-A Multicenter, Multinational, Before-and-After Intervention Trial. Crit Care Med. 2020;48(2). https://doi.org/10.1097/CCM.0000000000004113 15. Kalfon P, Alessandrini M, Boucekine M, Renoult S, Geantot M-A, Deparis-Dusautois S, et al. Tailored multicomponent program for discomfort reduction in critically ill patients may decrease post-traumatic stress disorder in general ICU survivors at 1 year. Intensive Care Med. 2019;45(2):223-35. https://doi.org/10.1007/s00134-018-05511-y 16. GarrousteOrgeas M, Flahault C, Vinatier I, Rigaud JP, Thieulot-Rolin N, Mercier E, et al. Effect of an ICU Diary on Posttraumatic Stress Disorder Symptoms Among Patients Receiving Mechanical Ventilation: A Randomized Clinical Trial. JAMA. 2019;322(3):229-239. https://doi.org/10.1001/jama.2019.9058 17. Jensen JF, Egerod I, Bestle MH, Christensen DF, Elklit A, Hansen RL, et al. A recovery program to improve quality of life, sense of coherence and psychological health in ICU survivors: a multicenter randomized controlled trial, the RAPIT study. Intensive Care Med. 2016;42(11):1733-43.

https://doi.org/10.1007/s00134-016-4522-1 18. Ostacher MJ, Cifu AS. Management of Posttraumatic Stress Disorder. JAMA. 2019;321(2):200-201. https://doi.org/10.1001/jama.2018.19290 19. American Psychological Association. Clinical Practice Guideline for the Treatment of Posttraumatic Stress Disorder (PTSD) in Adults, 2017. Online access: https://www.apa.org/ptsd-guideline/ptsd.pdf 20. Gawlytta R, Niemeyer H, Böttche M, Scherag A, Knaevelsrud C, Rosendahl J. Internet-based cognitive-behavioural writing therapy for reducing post-traumatic stress after intensive care for sepsis in patients and their spouses (REPAIR): study protocol for a randomised-controlled trial. BMJ Open 2017;7:e014363. https://doi.org/10.1136/bmjopen-2016-014363 21. Weathers FW, Litz BT, Keane TM, Palmieri PA, Marx 
BP, Schnurr PP. The PTSD Checklist for DSM-5 (PCL-5). Scale available from the National Center for PTSD, 2013. Online access: http://www.ptsd.va.gov. 22. Weathers FW, Blake DD, Schnurr PP, Kaloupek DG, Marx BP, Keane TM. The Life Events Checklist for DSM-5 (LEC-5). Scale available from the National Center for PTSD, 2013. Online access: http://www.ptsd.va.gov. 23. Weathers FW, Blake DD, Schnurr PP, Kaloupek DG, Marx BP, Keane TM. The Clinician-Administered PTSD Scale for DSM-5 (CAPS-5). Scale available from the National Center for PTSD, 2013. Online access: http://www.ptsd.va.gov. 24. Wittchen HU, Zaudig M, Fydrich T. [Structured Clinical Interview for DSM-IV Axis I Disorders (SCID-I)]. Göttingen: Hogrefe, 1997. 25. Krüger-Gottschalk A, Knaevelsrud C, Rau H, Dyer A, Schäfer I, Schellong J, et al. The German version of the posttraumatic stress disorder checklist for DSM-5 (PCL-5): psychometric properties and diagnostic utility. BMC Psychiatry 2017;17:379. https://doi.org/10.1186/s12888-017-1541-6 26. Blevins CA, Weathers FW, Davis MT, Witte TK, Domino JL. The posttraumatic stress disorder checklist for DSM-5 (PCL-5): development and initial psychometric evaluation. J Trauma Stress 2015;28:489-98. https://doi.org/10.1002/jts.22059 27. Derogatis LR. Brief Symptom Inventory (BSI) 18. Administration, scoring and procedures manual. Minneapolis, MN: NCS Pearson, 2000. 28. Franke GH, Jaeger S, Glaesmer H, Barkmann C, Petrowski K, Brähler E. Psychometric analysis of the brief symptom inventory 18 (BSI-18) in a representative German sample. BMC Med Res Methodol 2017;17(1):14.

https://doi.org/10.1186/s12874-016-0283-3 29. Hendrick SS. A Generic Measure of Relationship Satisfaction. J Marriage Fam 1988;50:93-98. 30. Hassebrauck M. [ZIP - A scale for assessment of satisfaction in close relationships]. Z Sozpsychol 1991;22:256-259. 31. Herdman M, Gudex C, Lloyd A, Janssen $M$, Kind P, Parkin D, et al. Development and preliminary testing of the new five-level version of EQ-5D (EQ-5D-5L). Qual Life Res 2011;20:1727-1736. 32. Ludwig K, Graf von der Schulenburg JM, Greiner W. German Value Set for the EQ-5D-5L. Pharmacoeconomics. 2018 Jun;36(6):663-674.

https://doi.org/10.1007/s40273-018-0615-8 33. Küster A, Niemeyer H, Knaevelsrud C. Internet-based interventions for posttraumatic stress: A meta-analysis of randomized controlled trials. Clin Psychol Rev 2016;43:1-16 34. van Buuren S, Groothuis-Oudshoorn K. mice: Multivariate Imputation by Chained Equations in R. Journal of Statistical Software 2011;45(3):1-67. https://doi.org/10.18637/jss.v045.i03 35. Kosilek RP, Baumeister SE, Ittermann T, Gründling M, Brunkhorst FM, Felix SB, et al. The association of intensive care with utilization and costs of outpatient healthcare services and quality of life. PLoS ONE. 2019;14(9):e0222671. https://doi.org/10.1371/journal.pone.0222671 36. Gawlytta R, Brunkhorst F, Niemeyer H, Boettche M, Knaevelsrud C, Rosendahl J. Dyadic post-traumatic stress after intensive care: Case report of a sepsis patient and his wife. Intensive Crit Care Nurs. 2020;58:102806.

https://doi.org/10.1016/j.iccn.2020.102806

\section{Figures}




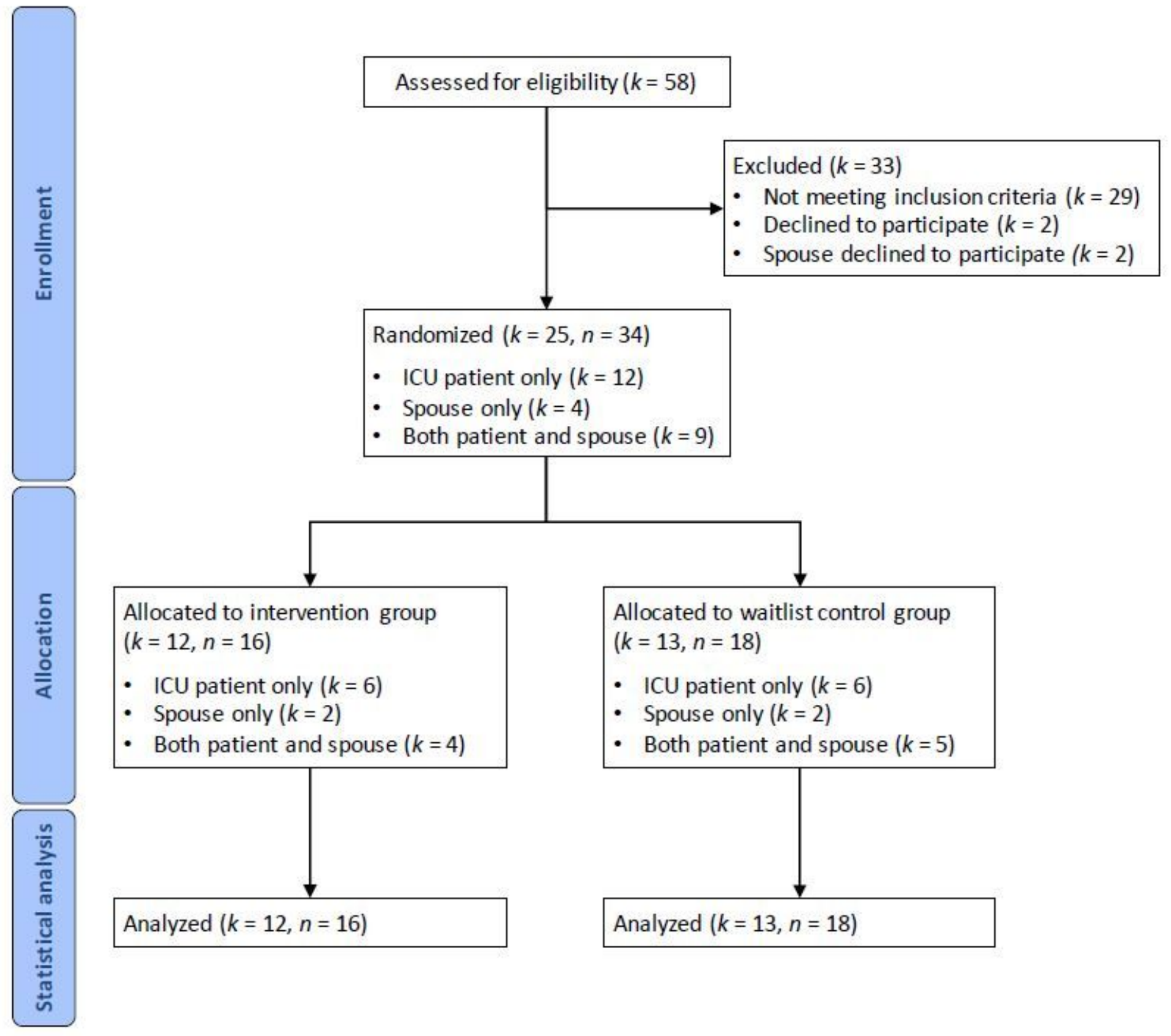

Figure 1

Flow diagram. Note: The number of dyads $(k)$ and the number of participants with PTSD symptoms ( $n$ ) are provided. Reasons for exclusions are given. Abbreviations: ICU, intensive care unit; PTSD, posttraumatic stress disorder. 
ICBT group (post-ICU patient)

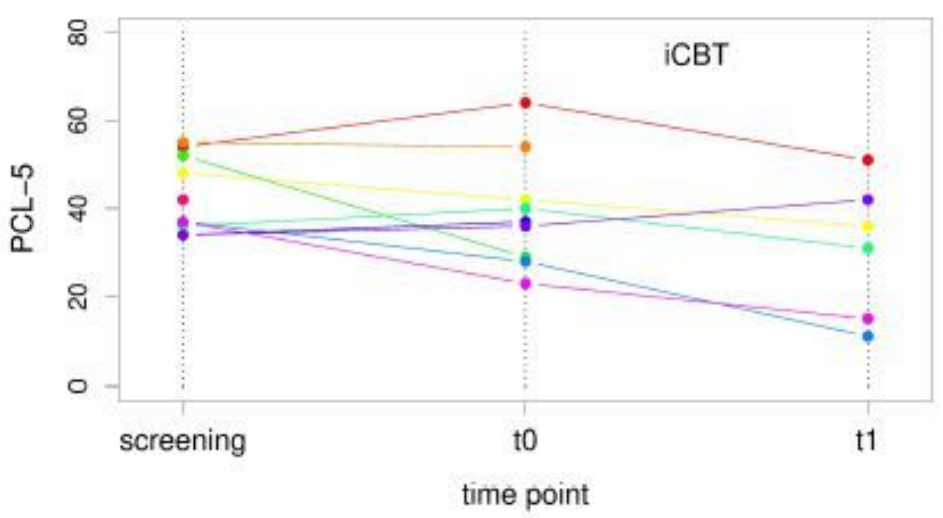

ICBT group (spouse)

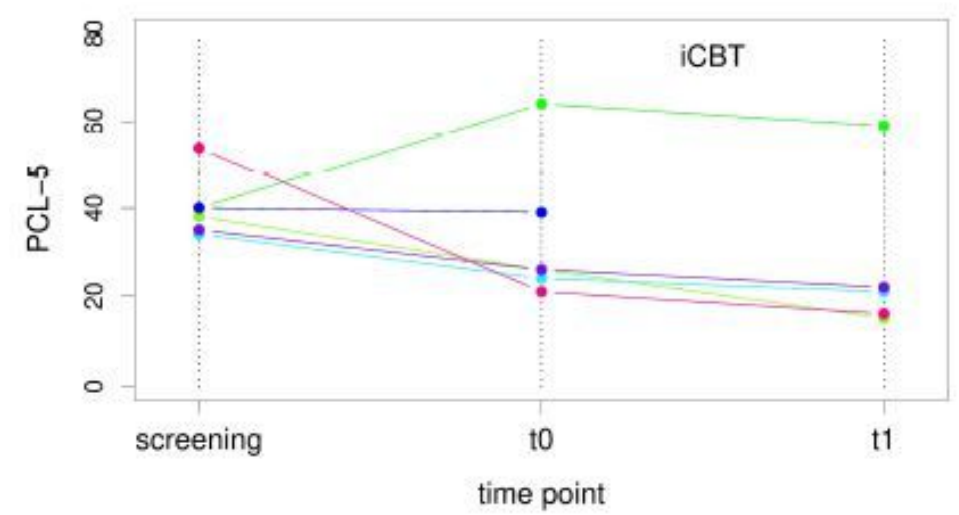

WL control group (post-ICU patient)

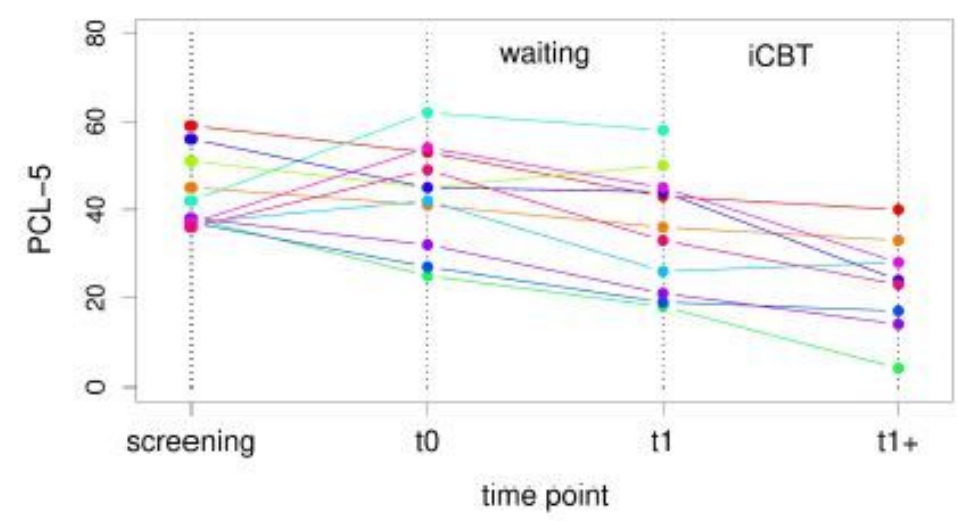

WL control group (spouse)

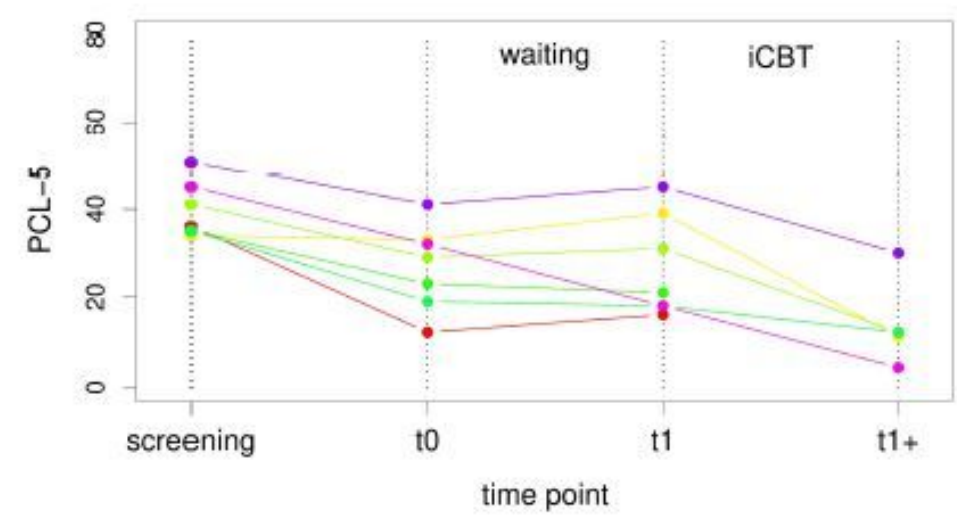

\section{Figure 2}

Observed PTSD symptoms (PCL-5 total score) in participants at trial assessments - stratified by post-ICU patient and his/her spouse as well as by treatment group (iCBT / WL control group). Note: Per treatment condition, dyad membership is color-coded. Higher PCL-5 scores indicate more severe symptoms. Note that one participant (former ICU patient in iCBT group) dropped-out directly after randomization. Values are provided for several time points (including approximately time specifications): screening (t0 - 4 weeks); t0, start of intervention (iCBT group) / waiting (WL control group); t1 (t0 + 5 weeks), end of intervention (iCBT group) / waiting (WL control group). In the WL control group, the end of intervention is at $\mathrm{t} 1+(\mathrm{t} 1+5$ weeks). Abbreviations: iCBT, internet-based cognitive-behavioral writing therapy; ICU, intensive care unit; PCL-5, PTSD Checklist for DSM-5; PTSD, post-traumatic stress disorder; WL, waitlist.

\section{Supplementary Files}

This is a list of supplementary files associated with this preprint. Click to download.

- REPAIRresultsSupplement1.docx

- REPAIRresultsSupplement2.docx 
- REPAIRgraphicalabstract.pdf

Page 19/19 